\title{
False-negative MRI biomarkers of tumour response to targeted cancer therapeutics
}

\author{
JKR Boult ${ }^{1,3}$, Y Jamin ${ }^{1,3}$, V Jacobs ${ }^{2}$, LD Gilmour ${ }^{1,4}$, S Walker-Samuel ${ }^{1,5}$, J Halliday², P Elvin ${ }^{2}$, AJ Ryan ${ }^{2,6}$, \\ JC Waterton ${ }^{2}$ and SP Robinson ${ }^{*, 1}$
}

'Cancer Research UK and EPSRC Cancer Imaging Centre, Division of Radiotherapy and Imaging, The Institute of Cancer Research and Royal Marsden NHS Foundation Trust, Sutton, Surrey SM2 5NG, UK; ${ }^{2}$ AstraZeneca, Alderley Park, Cheshire SKIO 4TG, UK

BACKGROUND: Non-invasive quantitative imaging biomarkers are essential for the evaluation of novel targeted therapeutics. Before deployment in clinical trials, such imaging biomarkers require qualification, typically through pre-clinical identification of imaging-pathology correlates.

METHODS: First, in investigating imaging biomarkers of invasion, the response of orthotopic murine PC3 prostate xenografts to the Src inhibitor saracatinib was assessed using susceptibility contrast MRI. Second, the longitudinal response of chemically induced rat mammary adenocarcinomas to the VEGFR2 inhibitor vandetanib was monitored by intrinsic susceptibility MRI, to identify the time window of transient vascular normalisation.

RESULTS: No significant differences in fractional blood volume (\%), vessel calibre $(\mu \mathrm{m})$, native $T_{1}$ (ms) or apparent water diffusion coefficient were determined, despite reduced expression of activated Fak and paxillin in the saracatinib cohort. Treatment with vandetanib elicited a 60\% antitumour response $(P<0.01)$, 80\% inhibition in vessel density $(P<0.05)$ and reduction in hypoxia $(P<0.05)$. There was, however, no significant change in tumour baseline $R_{2} *\left(\mathrm{~s}^{-1}\right)$ or carbogen-induced $\Delta R_{2} *$ with treatment. CONCLUSION: Reporting negative imaging biomarker responses is important, to avoid the risk of clinical trials using the same biomarkers being undertaken with a false expectation of success, and the abandonment of promising new therapeutics based on a false-negative imaging biomarker response being mistaken for a true-negative.

British Journal of Cancer (2012) 106, 1960-1966. doi:I0.1038/bjc.2012.208 www.bjcancer.com

Published online 17 May 2012

(C) 2012 Cancer Research UK

Keywords: MRl; biomarkers; Src; VEGF

Anti-cancer drug discovery and development is now predominantly targeted to cancer-causing genes and associated pathways. For the evaluation of novel oncology therapeutics, the use of pharmacodynamic biomarkers, which may include imaging biomarkers, is now essential (Workman et al, 2006; Waterton and Pylkkanen, 2012). For example, contrast-enhanced magnetic resonance imaging, as well as the established biomarkers it affords, is now routinely deployed in early clinical trials, and has proven particularly powerful for the assessment of tumour vascular-targeting agents (Leach et $a l, 2005)$. The new generation of targeted cancer therapeutics requires the development and evaluation of additional imaging biomarkers that detect anticancer activity, based on the targeted mechanism of action. Imaging biomarkers for assessing

\footnotetext{
*Correspondence: Dr SP Robinson; E-mail: Simon.Robinson@icr.ac.uk

${ }^{3}$ These authors contributed equally to this work.

${ }^{4}$ Current address: Translational Radiation Biology, The Beatson Institute for Cancer Research, Bearsden, Glasgow, UK

${ }^{5}$ Current address: UCL Centre for Advanced Biomedical Imaging, Department of Medicine and Institute of Child Health, University College London, 72 Huntley Street, London WCIE 6DD, UK

${ }^{6}$ Current address: Gray Institute for Radiation Oncology \& Biology, University of Oxford, Old Road Campus Research Building, Roosevelt Drive, Headington, Oxford, UK

Received 22 February 2012; accepted 19 April 2012; published online 17 May 2012
}

tumour pathophysiology and response require evaluation before they can be deployed in clinical trials. In particular, the evaluation of imaging-pathology correlation, and whether changes in the imaging biomarker reflect desired changes in the underlying pathology, is extremely important, but can often only meaningfully be studied in animal models. However, while good registries exist for clinical trials, there is no equivalent for animal studies, so if investigators fail to publish "negative" findings in animals (creating publication bias), there is a risk that clinical trials using imaging biomarkers may be undertaken with a false expectation of success, or even that promising investigational drugs may be abandoned, because a false-negative imaging biomarker response is mistaken for a true-negative. With this risk in mind, in this work we describe two apparently false-negative animal studies using MRI biomarkers of potential clinical utility.

Two pre-clinical studies were performed in which emerging non-invasive MRI biomarkers were correlated with histology to assess tumour response and target inhibition. Both studies used models and doses exhibiting antitumour efficacy, so that failure to elicit an imaging biomarker response in the presence of a histological response would suggest a false-negative for the imaging biomarker. In evaluating potential imaging biomarkers of tumour invasion, the first study focussed on the response to SrC inhibition. Numerous proteins are involved in the disruption of cell-cell and cell-extracellular matrix contact, and which facilitate cell migration, invasion and tumour progression. These include the Src kinases, a family of membrane-associated non-receptor 
tyrosine kinases expressed in a wide range of human cancers, the elevated expression of which is linked to a more invasive tumour phenotype and metastasis, and poor prognosis (Frame, 2002; Ishizawar and Parsons, 2004; Rusello and Shore, 2004). Inhibitors of Src are thus being developed as an anti-invasion strategy for the treatment of cancer, and the novel, oral, potent and selective inhibitor of Src, saracatinib, (AZD0530, AstraZeneca, Macclesfield, UK), has been shown to be active against a wide range of cancers both in vitro and in vivo (Green et al, 2009). Here the response of murine orthotopic PC3 prostate xenografts to saracatinib was investigated using a multi-parametric MRI approach, to assess any differences in tissue cellularity with treatment through quantification of the apparent diffusion coefficient (ADC) and native longitudinal MRI relaxation time $T_{1}$ (Walker-Samuel et al, 2009; McSheehy et al, 2010). Any potential anti-angiogenic effects were interrogated using susceptibility contrast MRI with intravascular ultrasmall superparamagnetic iron oxide (USPIO) particles, enabling steady-state determination of the tumour fractional blood volume (fBV, \%) and vessel size index (VSI, $\mu \mathrm{m})$, a weighted average measure of vessel calibre (Tropres et al, 2004; Walker-Samuel et al, 2012).

In the second study, the response of MNU-induced rat mammary adenocarcinomas to vascular endothelial growth factor (VEGF) signalling inhibition was investigated using intrinsic susceptibility MRI, in which image contrast relies on endogenous paramagnetic deoxyhaemoglobin that increases the MRI transverse relaxation rate $R_{2}{ }^{*}\left(\mathrm{~s}^{-1}\right)$ of water in blood and tissue surrounding blood vessels. Changes in tumour $R_{2}{ }^{*}$, induced by carbogen $\left(95 \% \mathrm{O}_{2} / 5 \% \mathrm{CO}_{2}\right)$ breathing, can be used to assess tumour vascular function (Howe et al, 1999; Robinson et al, 2003). Vascular endothelial growth factor is considered the most potent angiogenic growth factor, and mediates its effects principally via two receptor tyrosine kinases expressed on endothelial cells, Flt-1 (VEGFR1) and KDR/Flk-1 (VEGFR2). Vandetanib (ZD6474, CAPRELSA, AstraZeneca) is a low molecular weight inhibitor of KDR tyrosine kinase activity and VEGF-stimulated endothelial cell proliferation, shown to significantly inhibit tumour growth in a wide range of models in vivo, and has undergone Phase III clinical trials in nonsmall cell lung cancer and in patients with advanced or metastatic medullary thyroid carcinoma (Wedge et al, 2002). We hypothesised that temporal changes in $R_{2}{ }^{*}$ and carbogen-induced $\Delta R_{2}{ }^{*}$ following treatment with vandetanib could be used to identify the time window associated with any therapy-induced transient vascular normalisation (Winkler et al, 2004).

\section{MATERIALS AND METHODS}

\section{Animals, tumours, anaesthesia and drug formulation}

All experiments were performed in accordance with the local ethical review panel, the UK Home Office Animals Scientific Procedures Act, 1986 and the UKCCCR guidelines (Workman et al, 2010). Orthotopic prostate tumours were propagated by injection of $5 \times 10^{5}$ PC3 human prostate carcinoma cells into the ventral prostate gland of male NCr nude mice (Sanderson et al, 2006). Female Sprague Dawley rats were injected with a single $37.5 \mathrm{mg} \mathrm{kg}^{-1}$ intraperitoneal dose of refrigerated $\mathrm{N}$-methyl- $\mathrm{N}$ nitrosourea (MNU, Sigma-Aldrich, Poole, UK), resulting in breast tumours that developed in various sites associated with the mammary tissue (McPhail and Robinson, 2010).

For MRI, animals were anaesthetised with either a $10-\mathrm{ml} \mathrm{kg}^{-1}$ (mice) or $4-\mathrm{ml} \mathrm{kg}^{-1}$ (rats) intraperitoneal injection of fentanyl citrate $\left(0.315 \mathrm{mg} \mathrm{ml}^{-1}\right)$ with fluanisone $\left(10 \mathrm{mg} \mathrm{ml}^{-1}\right.$, (Hypnorm; Janssen Pharmaceutical Ltd, High Wycombe, UK), midazolam ( $5 \mathrm{mg} \mathrm{ml}^{-1}$, Hypnovel; Roche, Burgess Hill, UK) and sterile water $(1: 1: 2)$. Core body temperature was monitored and maintained by warm air blown through the magnet bore.
Saracatinib was formulated in $0.5 \%$ hydroxypropyl methyl cellulose (Fluka, Poole, UK) and $0.1 \%$ polysorbate 80 (Fluka). Vandetanib was prepared with $1 \%$ polysorbate 80 (Fluka), diluted in sterile water, and milled overnight to generate a uniform suspension.

\section{Study 1 - multi-parametric MRI assessment of tumour response to saracatinib}

Mice bearing orthotopic prostate tumours, detected by palpation, were stratified to receive a daily oral dose of $25 \mathrm{mg} \mathrm{kg}^{-1}$ saracatinib $(n=7)$ or vehicle alone $(n=7)$ over 5 days. On the fifth day of treatment, mice were imaged 2 hours after the saracatinib dose. A lateral tail vein was cannulated with a $27-G$ butterfly catheter (Venisystems, Hospira, Royal Leamington Spa, UK) to enable the remote administration of USPIO particles. Each mouse was then positioned supine within a $3-\mathrm{cm}$ birdcage ${ }^{1} \mathrm{H}$ coil in a 7 -Tesla, horizontal bore microimaging system (Bruker Biospin, Ettlingen, Germany).

A morphological fast, multi-slice RARE spin-echo sequence was first used for both localisation of the tumour and subsequent determination of tumour volume. Multi gradient-echo (MGE), spin-echo (SE) and diffusion-weighted images were then acquired at an identical resolution (matrix size $64 \times 64$, FOV $3.3 \mathrm{~cm} \times 3.3 \mathrm{~cm}$ ) to quantify $R_{2}{ }^{*}, R_{2}$ and ADC from a single central transverse $1-\mathrm{mm}$ thick slice. Multi gradient-echo images were acquired with 8 echoes ( $\mathrm{TE}=6.1$ to $28.2 \mathrm{~ms}$, $\mathrm{TR}=300 \mathrm{~ms}$, flip angle $=45^{\circ}$ and 8 averages). A first SE image was acquired with $\mathrm{TR}=3000 \mathrm{~ms}$, flip angle $=90^{\circ}$ and 12 averages and $\mathrm{TE}=8 \mathrm{~ms}$, followed by the acquisition of a second SE image with $\mathrm{TE}=80 \mathrm{~ms}$. Diffusion data were acquired using a diffusion-weighted SE sequence with $6 \mathrm{~b}$-values ranging from 40 to $1000 \mathrm{~s} \mathrm{~mm}^{-2}$, gradient duration $=5 \mathrm{~ms}$, gradient spacing $=25 \mathrm{~ms}$, $T R=1000 \mathrm{~ms}$, $\mathrm{TE}=36 \mathrm{~ms}, 1$ average). In addition, native $T_{1}$ was quantified using an inversion recovery (IR) True-FISP sequence with 8 segments, 50 inversion times $(\mathrm{TI}=25-1450 \mathrm{~ms}), \mathrm{TE}=1.2 \mathrm{~ms}, \mathrm{TR}=2.5 \mathrm{~ms}$, and a total scan $\mathrm{TR}=10 \mathrm{~s}$ and 8 averages (matrix size $128 \times 96$ ). Following acquisition of these images, $200 \mu \mathrm{mol} \mathrm{kg}^{-1}$ USPIO particles (Sinerem, ferumoxtran-10, Guerbet, Roissy, France) in saline was injected intravenously and allowed to circulate for 2 minutes to equilibrate, after which a second set of MGE, SE and IR-True-FISP images were acquired. The MGE, SE and ADC data were used to generate maps and quantify tumour fBV and VSI as previously described (Walker-Samuel et al, 2012).

To validate the fBV and VSI measurements, Hoechst 33342, a fluorescent endothelial stain of perfused blood vessels, was administered as a terminal experiment following the MRI measurements. Following the imaging session, $15 \mathrm{mg} \mathrm{kg}^{-1}$ Hoechst 33342 (Sigma, Poole, UK) was administered i.v. and 1 minute later, mice were killed, tumours rapidly excised, and cut in half, with one half rapidly frozen over liquid nitrogen, and the other half fixed in $10 \%$ formalin. Serial $10 \mu \mathrm{m}$ frozen tissue sections were cut, and slides fixed in ice cold acetone for $10 \mathrm{~min}$ before being imaged on a fluorescence microscope for Hoechst 33342 uptake. Fluorescence signals from whole tumour sections were then recorded at $365 \mathrm{~nm}$ using a motorised scanning stage (Prior Scientific Instruments, Cambridge, UK) attached to a BX51 microscope (Olympus Medical, Southend-on-Sea, UK) driven by CellP (Soft Imaging System, Munster, Germany). The area of the tumour section with Hoechst 33342 fluorescence was determined on whole tumour composite images and expressed as a percentage of the whole tumour section (mean Hoechst perfused area) (Walker-Samuel et al, 2012). Src pathway inhibition was assessed with immunohistochemical biomarker analysis of activated Src (clone 28 antibody, Invitrogen, Paisley, UK), total (C-903, Santa Cruz, Heidelberg, Germany) and phosphorylated (\#44-6261-0300, Biosource UK Ltd, London, UK) focal adhesion kinase (Fak), and total (H-114, Santa Cruz) and phosphorylated (\#22-5922-08, 
Biosource UK Ltd) paxillin, on formalin fixed paraffin embedded sections, as previously described (Green et al, 2009).

\section{Study 2 - tumour response to vandetanib assessed by intrinsic susceptibility MRI}

On day 0, rat tumour volume was measured using callipers, assuming an ellipsoidal shape, and the formula: tumour volume $=\pi / 6$.(length $\times$ width $\times$ depth). Rats were then positioned supine within a $64-\mathrm{mm}$ i.d. birdcage coil in the 7-Tesla magnet, and a nosepiece equipped with a scavenger positioned for delivery of air and carbogen gas. To quantify tumour $R_{2}{ }^{*}$ while the rat breathed air, MGE images were acquired from three $1.56 \mathrm{~mm}$ thick transverse slices through the tumour, using 8 averages of 128 phase-encoding steps over a $6 \times 6-\mathrm{cm}$ field of view, giving a temporal resolution of $3.5 \mathrm{~min}$. Images were acquired using a train of 8 echoes spaced $3.14 \mathrm{~ms}$ apart, initial $\mathrm{TE}=6.21 \mathrm{~ms}$, flip angle $\alpha=45^{\circ}$ and $\mathrm{TR}=200 \mathrm{~ms}$. The gas supply was then switched to carbogen at $21 / \mathrm{min}$ and, following a brief transition time of typically $2 \mathrm{~min}$, a second set of MGE images were acquired again from the same slice positions as during air breathing.

The rats were allowed to recover, and then subsequently given a daily dose of either $30 \mathrm{mg} \mathrm{kg}^{-1}$ vandetanib p.o., or vehicle alone, over 4 days, during which tumour volume was also measured. Intrinsic susceptibility MRI was repeated on day 2 and day 4 .

After the final MRI session on day $4,60 \mathrm{mg} \mathrm{kg}^{-1}$ of the hypoxia marker pimonidazole (Hypoxyprobe Inc., Burlington, USA) was administered intraperitoneally. After $45 \mathrm{~min}, 15 \mathrm{mg} \mathrm{kg}^{-1}$ Hoechst 33342 was injected via a lateral tail vein, and, 1 min later, tumours rapidly excised and cut in half, with one half rapidly frozen over liquid nitrogen, and the other half fixed in $10 \%$ formalin.

For immunofluorescence, frozen $10 \mu \mathrm{m}$ tissue sections were cut from at least 3 locations from each tumour half, and slides imaged on a fluorescence microscope for Hoechst 33342 uptake, as described earlier. The slides were then blocked with $2 \%$ bovine serum albumin (w/v) (Vector Laboratories, Peterborough, UK) and $5 \%$ goat serum albumin (v/v) (Sigma-Aldrich) in PBS for $30 \mathrm{~min}$, followed by incubation for $2 \mathrm{~h}$ with a FITC-conjugated mouse anti-pimonidazole antibody protected from light (Hypoxyprobe Inc., 1:500). The slides were then washed and imaged at $450-490 \mathrm{~nm}$ using the same stage co-ordinates as used for the Hoechst 33342 image, and pimonidazole adduct formation quantified as previously described (Boult et al, 2011). Vascular endothelial growth factor signalling inhibition was immunohistochemically assessed on formalin fixed paraffin embedded sections, using a rabbit monoclonal antibody against VEGFR2 (\#2479, Cell Signalling Technology, Leiden, The Netherlands). Tissue sections were imaged using a ScanScope digital scanner (Aperio, Oxford, UK), regions of interest identified for analysis to exclude areas of background staining or poor sample quality, and the mean vessel density (MVD) per $\mathrm{mm}^{2}$ was assessed using the microvessel analysis algorithm running under ImageScope (Aperio).

\section{RESULTS}

\section{Study 1}

There was no significant difference in tumour volume between the cohort treated with saracatinib $\left(632 \pm 99 \mathrm{~mm}^{3}\right)$ and the vehicle control $\left(552 \pm 114 \mathrm{~mm}^{3}\right)$ cohort after 5 days treatment. Representative parametric MRI maps determined from a vehicle-treated mouse and a mouse treated with $25 \mathrm{mg} \mathrm{kg}^{-1}$ saracatinib daily, over 5 days, are shown in Figure 1. From both the fBV and VSI maps, it was evident that uptake of the USPIO particles was typically restricted to the tumour periphery. Both ADC and native $T_{1}$ were more homogeneously distributed across the tumours. The quantitative MRI data are summarised in Table 1. No statistically significant differences were found between any of the quantitative MRI parameters between the vehicle and saracatinib-treated cohorts.

Table I Summary of the quantitative MRI data from Study I

\begin{tabular}{lcr}
\hline & Vehicle & Saracatinib \\
\hline Volume $\left(\mathrm{mm}^{3}\right)$ & $552 \pm 114$ & $632 \pm 99$ \\
ADC $\left(\times 10^{-6} \mathrm{~mm}^{2} \mathrm{~s}^{-1}\right)$ & $605 \pm 48$ & $620 \pm 28$ \\
Native $T_{1}(\mathrm{~ms})$ & $1999 \pm 41$ & $2016 \pm 42$ \\
fBV $\%)$ & $4.08 \pm 1$ & $4.46 \pm 1$ \\
VSI $(\mu \mathrm{m})$ & $52 \pm 11$ & $51.2 \pm 8$ \\
\hline
\end{tabular}

Tumour volume, apparent diffusion coefficient (ADC), native longitudinal relaxation time $T_{1}$, fractional blood volume ( $\mathrm{FBV}$ ) and vessel size index (VSI), determined from orthotopically propagated murine PC3 prostate xenografts after 5-days treatment with a daily oral dose of vehicle or $25 \mathrm{mg} \mathrm{kg}^{-1}$ saracatinib. Data are mean \pm I s.e.m, $n=7$ per treatment arm
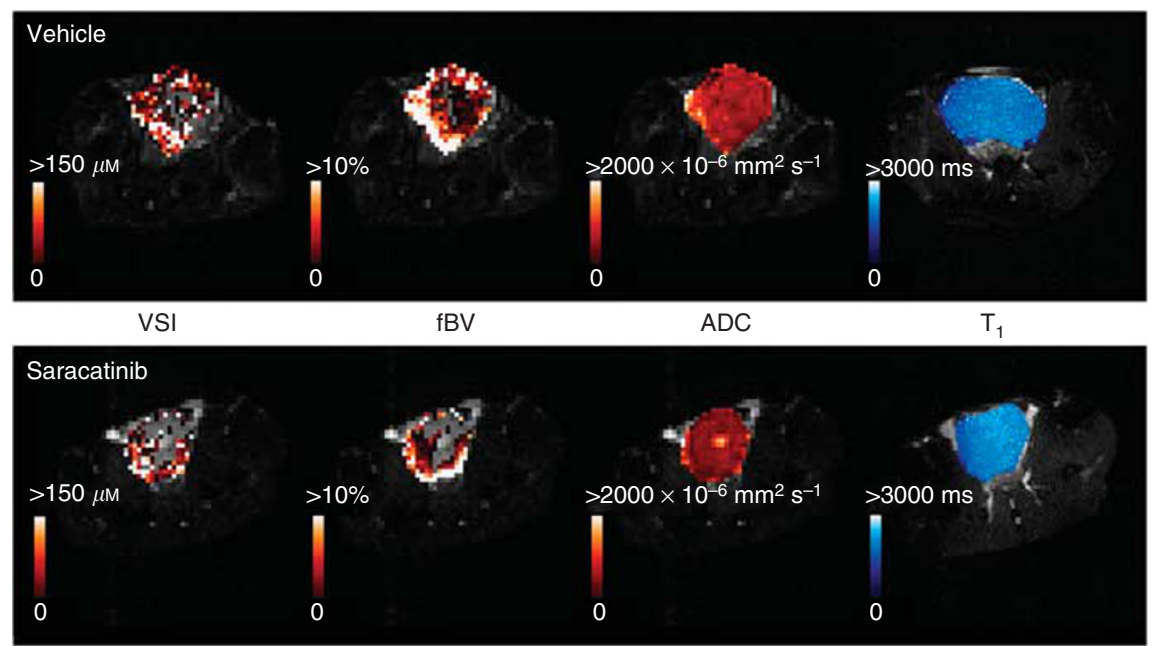

Figure I Representative parametric MRI maps of vessel size index (VSI), fractional blood volume (fBV), apparent diffusion coefficient (ADC) and

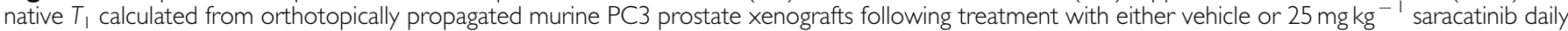
over 5 days. 
There was no difference in the mean Hoechst 33342 perfused area between the saracatinib-treated cohort $(5.14 \pm 0.9 \%)$ and vehicle control ( $4.84 \pm 0.8 \%$, Figure $2 \mathrm{~A}$ and $\mathrm{B})$. Immunohistochemical analysis showed a slight increase in levels of active Src in the tumours from the saracatinib-treated animals. A significant reduction in the expression levels of phosphorylated Fak (pFak) Y861 and phosphorylated Paxillin (pPaxillin) Y31 was observed at the membrane in the saracatinib-treated tissue (Figure 2C). Staining in the nuclei of tumours from the treated animals was increased, indicating relocation of $\mathrm{pFak}$ and pPaxillin. No change was seen in the total expression of Fak and Paxillin.

\section{Study 2}

Anatomical $T_{2}$-weighted MR images acquired from one MNUinduced rat mammary tumour before and during treatment with vandetanib are shown in Figure 3A. Highly significant antitumour activity was observed in all of the vandetanib-treated animals, whereas tumours in vehicle-treated rats progressed (Figure 3B). Treatment with vandetanib induced a significant $17 \pm 6 \%$ antitumour response at day 2 , reaching a highly significant $60 \pm 6 \%$ reduction in normalised tumour volume after 4 days treatment (Figure $3 \mathrm{C}$ ).
The overall mean baseline tumour $R_{2}{ }^{*}$ at day 0 was $103.9 \pm 15 \mathrm{~s}^{-1}$. Irrespective of treatment, there was no significant difference in tumour $R_{2}{ }^{*}$ over 4 days (vehicle, $101.1 \pm 17$ to $126.6 \pm 20 \mathrm{~s}^{-1}(n=4)$; vandetanib, $110.8 \pm 27$ to $117.1 \pm 32 \mathrm{~s}^{-1}(n=4)$. No clear pattern of baseline $R_{2}{ }^{*}$ or carbogen-induced $\Delta R_{2}{ }^{*}$ response was evident either in the regressing tumours treated with vandetanib, or in the vehicle controls, over 5 days (data not shown). Composite fluorescence images of Hoechst 33342 uptake and pimonidazole adduct formation in tumour sections obtained from a vehicle and vandetanib-treated rat are shown in Figure 4A. Both histological markers were heterogeneously distributed across the tumours. While there was no significant difference in tumour perfusion, treatment with vandetanib resulted in a clear and significant reduction in hypoxia compared with vehicle control (Figure 4B). Images from tumour tissue sections from vehicle and vandetanib-treated rats immunohistochemically stained for VEGFR2 are shown in Figure 4C. Quantification of VEGFR2 MVD revealed a significant $\sim 80 \%$ inhibition in the vandetanib-treated rats (Figure 4D).

\section{DISCUSSION}

Within this era of novel targeted cancer therapeutics has come the associated need to evaluate and qualify specific imaging
A
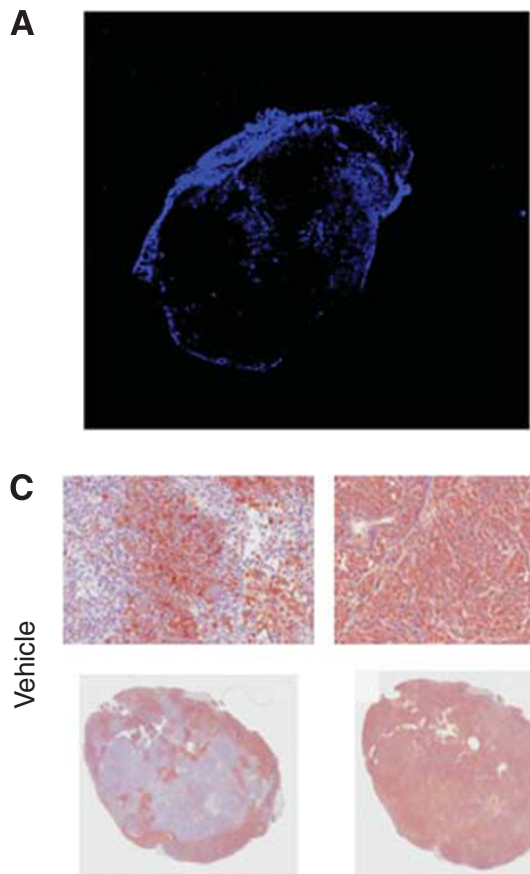

pFak Y861
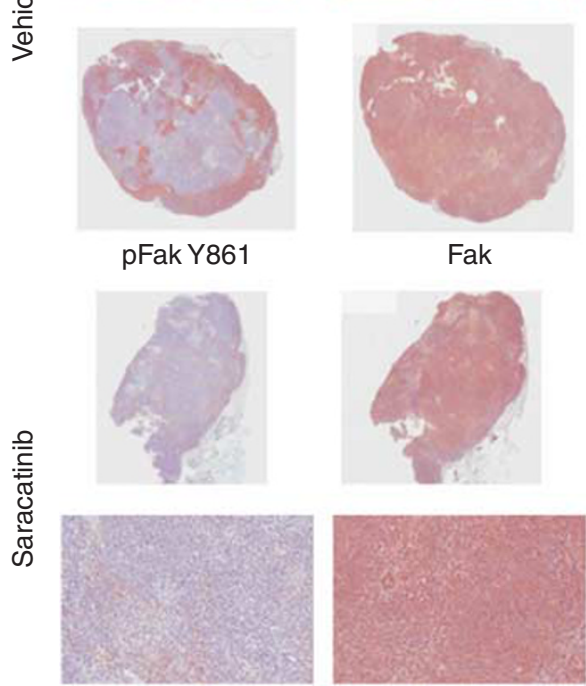

Fak

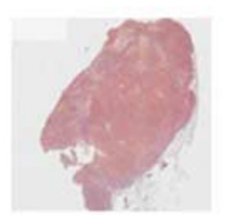

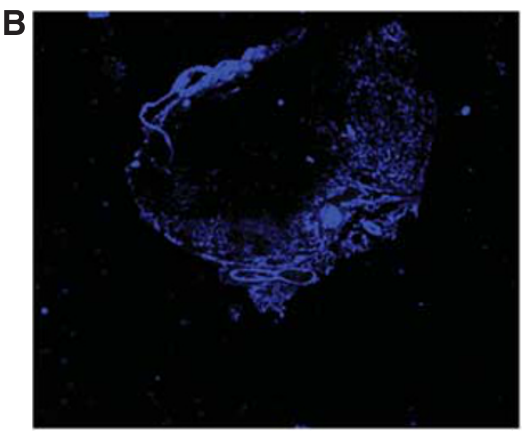
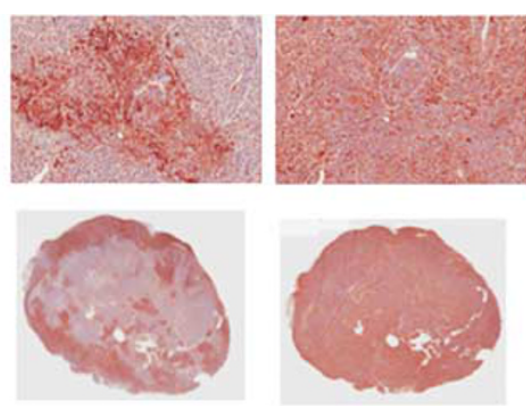

pPaxillin Y31
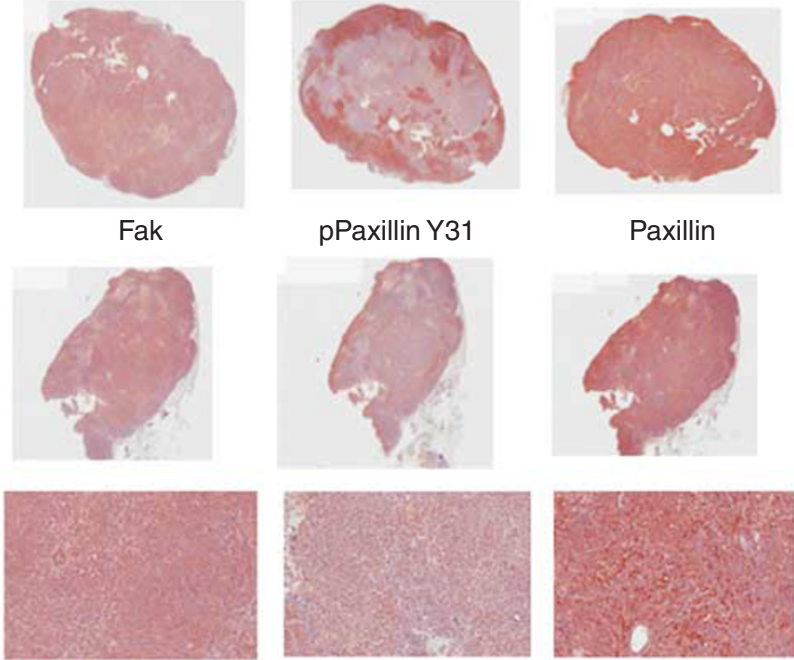

Paxillin
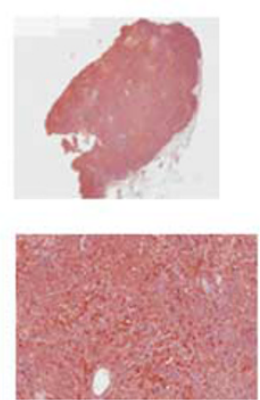

Figure 2 Representative composite fluorescence images of the uptake of the perfusion marker Hoechst 33342 in whole tumour sections from orthotopic PC3 prostate xenografts treated with $(\mathbf{A})$ vehicle or $(\mathbf{B}) 25 \mathrm{mg} \mathrm{kg}^{-1}$ saracatinib daily over 5 days. The mean Hoechst 33342 perfused area was $5.14 \pm 0.9 \%$ and $4.84 \pm 0.8 \%$ for the saracatinib-treated and vehicle cohorts, respectively, and there was no significant difference between them. (C) Representative high power and whole section images showing immunohistochemical staining for pFak Y86I, Fak, pPaxillin Y3I and paxillin in orthotopic PC3 prostate xenografts from mice treated with vehicle or $25 \mathrm{mg} \mathrm{kg}^{-1}$ saracatinib daily over 5 days. 

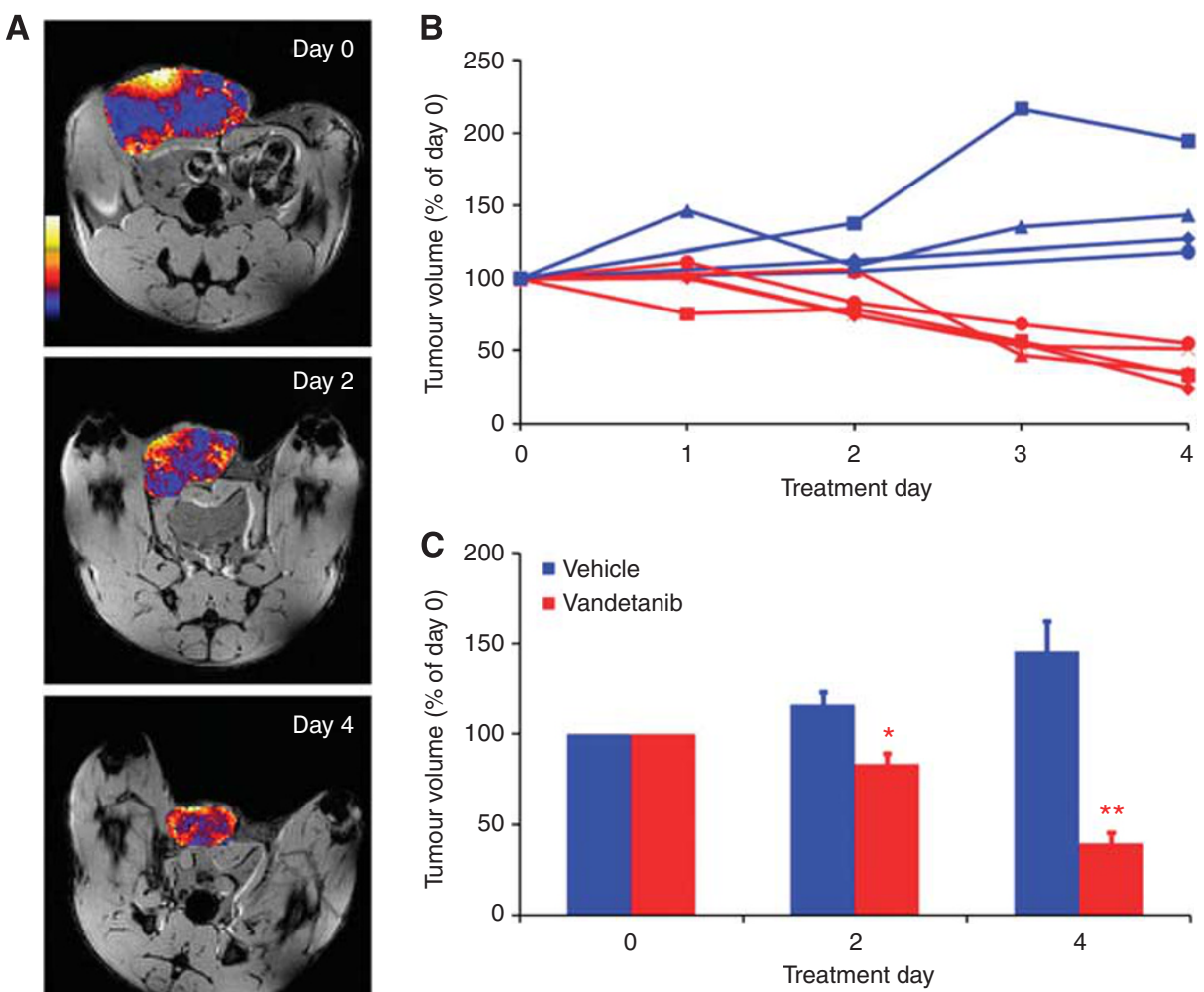

Figure 3 (A) Parametric $R_{2} *$ maps $\left(0-250 \mathrm{~s}^{-1}\right)$ overlaid on axial $T_{2}$-weighted $M R$ images acquired from one MNU-induced rat mammary carcinoma before and during treatment with $30 \mathrm{mg} \mathrm{kg}^{-1}$ vandetanib daily p.o. (B) Normalised tumour volumes from individual MNU-induced rat mammary carcinomas treated with either vandetanib (red, $n=5$ ) or vehicle (blue, $n=4$ ) p.o. over 5 days, and $(\mathbf{C})$ a summary of the differences in tumour volume between the cohorts on day 2 and 4 . Data are mean \pm I s.e.m. $* P<0.05$, $* * P<0.0$ I compared with day 0 , Student's paired $t$-test.

biomarkers of target inhibition that ultimately could be translated into the clinic. Many of these novel agents are not predicted to elicit dramatic tumour regressions when used as a monotherapy, although tumour growth may be delayed. Thus, to evaluate these agents successfully, more sophisticated ways of quantifying tumour response are required. Typically, non-invasive imaging is used to determine anatomical tumour size, based on the response evaluation criteria in solid tumours. Increasing evidence highlights that novel targeted agents can elicit efficacy before any reduction in tumour volume, and thus the measurement of imaging biomarkers of acute functional changes in tumour pathophysiology in vivo, such as those afforded by MRI, is more revealing (Michaelis and Ratain, 2006). A current challenge is the identification of appropriate imaging biomarker(s) to assess pharmacologically targeted inhibition, and which can potentially be used in a clinical trial setting (Waterton and Pylkkanen, 2012). Here we describe two pre-clinical MRI studies designed to investigate imaging biomarkers of response to Src and VEGF-signalling inhibition.

\section{Study 1}

In the first study, the response of an orthotopic PC3 orthotopic murine model to the Src kinase inhibitor saracatinib was assessed using a multi-parametric quantitative MRI approach. Elevated Src kinase activity in tumours reduces cell adhesion, enhances cell motility, and thus promotes an invasive phenotype (Ishizawar and Parsons, 2004; Rusello and Shore, 2004). Through its interaction with the integrin-binding non-receptor tyrosine kinase Fak, and the recruitment of cytoskeletal proteins such as paxillin, Src activity promotes cytoskeletal re-organisation, disruption of cell-cell adhesion and modulation of cell-matrix adhesion, facilitating epithelial-mesenchymal transition (EMT) and a motile cell phenotype (Frame, 2002). In considering imaging biomarkers of invasion, we hypothesised that inhibition of Src kinase activity would promote cell adhesion, resulting in increased restriction of tissue water mobility, and thus a reduction in tumour ADC. This hypothesis was not supported by the data, as saracatinib had no effect on MRI biomarkers of cellularity of size-matched orthotopically propagated PC3 prostate xenografts, as assessed by tumour ADC and native $T_{1}$. Despite this, immunohistochemical analysis clearly revealed that saracatinib was biologically active, as shown by the decrease in both FAK and paxillin phosphorylation, direct substrates of active Src kinase. Thus, in a setting when a treatment has antitumour efficacy and elicited the expected molecular response, but the biomarker failed to show the expected change, we would describe this as a false-negative imaging biomarker response.

Src activity has also been implicated in tumour angiogenesis (Gray et al, 2005; Lesslie et al, 2006). Herein, susceptibility contrast MRI was performed to investigate any potential anti-angiogenic effects of saracatinib treatment, but revealed no significant difference in $\mathrm{ABV}$ or VSI between the treated and control cohorts. The absence of any significant difference in Hoechst 33342 uptake provides good qualification of the non-invasively derived $\mathrm{fBV}$ data. In addition, the VSI of $\sim 50 \mu \mathrm{m}$ determined in the control cohort is in good agreement with that recently reported in this tumour model (Walker-Samuel et al, 2012). In this case, the treatment elicited neither the expected molecular response nor the expected biomarker change, so we would describe this as a true-negative imaging biomarker response. Together, these data suggest that saracatinib has little effect on tumour vasculature, corroborating other studies performed in a range of xenograft models (Green et al, 2009).

Thus, the non-invasive imaging biomarkers investigated in Study 1 were unable to reveal any acute differences in the 

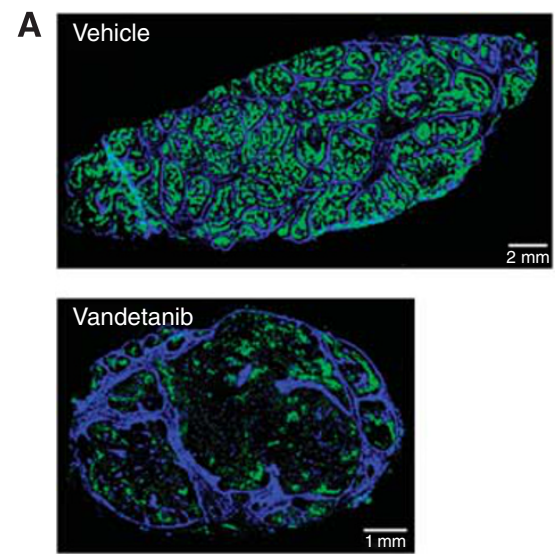

C

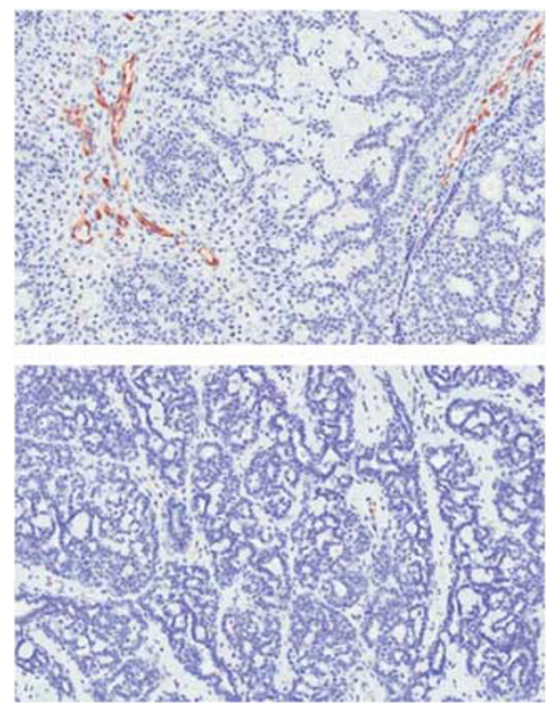

B

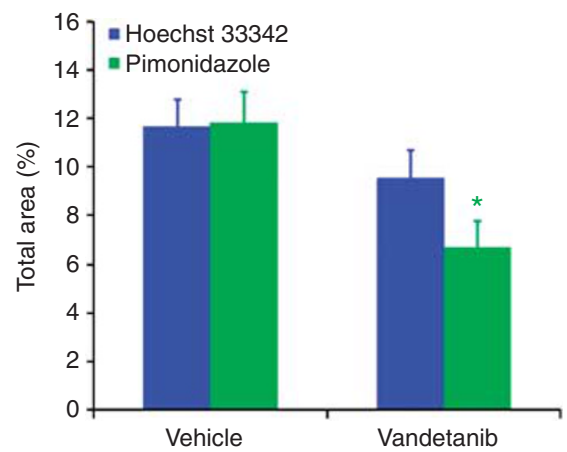

D

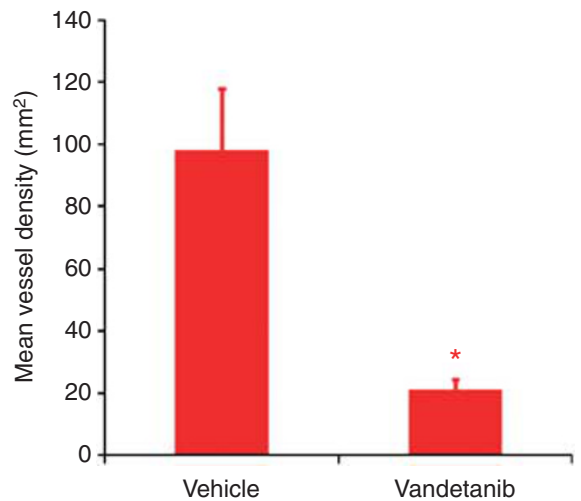

Figure 4 (A) Composite fluorescence images of whole tumour sections showing Hoechst 33342 uptake (blue) and pimonidazole adduct formation (green) from vehicle and vandetanib-treated rats. (B) Summary of the quantitative differences in Hoechst 33342 uptake and pimonidazole adduct formation determined between the vehicle $(n=4)$ and vandetanib-treated $(n=5)$ cohorts. Data are mean \pm 1 s.e.m. $* P<0.05$, Student's 2 -tailed $t$-test. (C) Microscopy images from localised regions of tissue sections immunohistochemically stained for VEGFR2 from a vehicle (upper panel) and vandetanibtreated (lower panel) rat. (D) Quantification of VEGFR2 mean vessel density from vehicle $(n=3)$ and vandetanib-treated $(n=3)$ animals. Data are mean \pm I s.e.m. $* P<0.05$, Student's 2-tailed t-test.

phenotype of orthotopically propagated PC3 prostate xenografts following five days treatment with saracatinib, despite clear immunohistochemical evidence of Src inhibition.

\section{Study 2}

The second study was concerned with longitudinally monitoring the response of MNU-induced rat mammary carcinomas to daily treatment with the VEGF signalling inhibitor vandetanib by intrinsic susceptibility MRI. Quantification of $R_{2}{ }^{*}$, sensitive to changes in paramagnetic deoxyhaemoglobin, has been exploited both pre-clinically and clinically as an emerging imaging biomarker of response to vascular targeted therapies, including vandetanib (Robinson et al, 2005; Mross et al, 2009). The ability of anti-angiogenic agents such as vandetanib to typically elicit modest tumour growth delay has been well documented in numerous pre-clinical studies. Given this, we hypothesised that quantification of tumour $R_{2}{ }^{*}$ and carbogen-induced $\Delta R_{2}{ }^{*}$ could provide non-invasive biomarkers of disrupted functional vasculature following treatment, and potentially be used to delineate any vandetanib-induced time window of vascular normalisation, in the absence of any significant tumour growth delay. Treatment with vandetanib had highly significant antitumour activity against these chemically induced rat mammary tumours, coupled with a significant reduction in tumour hypoxia that, importantly, was associated with significant target inhibition. Similar antitumour responses to vandetanib have been reported in other pre-clinical breast tumour models, but the precise mechanism of action is unclear (Miller et al, 2003; Heffelfinger et al, 2004; Wedge et al, 2007). Treatment with vandetanib has also been shown to reduce tumour oxygen consumption in the absence of any vascular remodelling (Ansiaux et al, 2009). While clearly an exciting and unanticipated overall response, the absence of any pattern in $R_{2}{ }^{*}$ or carbogen-induced $\Delta R_{2}{ }^{*}$ highlight the challenges of identifying meaningful quantitative imaging response biomarkers in the presence of such prolific tumour shrinkage. In this case, the treatment has antitumour efficacy and elicited a pronounced molecular response, but the corresponding imaging biomarker failed to show the expected change. We would describe this as a false-negative imaging biomarker response.

\section{CONCLUSION}

It is important to draw a distinction between validated surrogate endpoints, and other biomarkers that have utility although not considered validated (Downing et al, 2001). In the latter case, use of biomarkers is only appropriate when the risk of false-negatives 
and false-positives can be objectively assessed, and we hope that publication of these examples will inform the debate.

\section{ACKNOWLEDGEMENTS}

We acknowledge the support received for The Institute of Cancer Research CR-UK and EPSRC Cancer Imaging Centre, in

\section{REFERENCES}

Ansiaux R, Dewever J, Grégoire V, Feron O, Jordan BF, Gallez B (2009) Decrease in tumor cell oxygen consumption after treatment with vandetanib (ZACTIMA ${ }^{\mathrm{TM}}$; ZD6474) and its effect on response to radiotherapy. Radiat Res 172: 584-591

Boult JKR, Walker-Samuel S, Jamin Y, Leiper JM, Whitley GSJ, Robinson SP (2011) Active site mutant dimethylarginine dimethylaminohydrolase 1 expression confers an intermediate tumour phenotype in C6 gliomas. J Pathol 225: 344-352

Downing GJ, Atkinson AJ, Colburn WA, DeGruttola VG, DeMets DL, Hoth DF, Oates JA, Peck CC, Schooley RT, Spilker BA, Woodcock J, Zeger SL (2001) Biomarkers and surrogate endpoints: Preferred definitions and conceptual framework. Clin Pharmacol Ther 69: 89-95

Frame MC (2002) Src in cancer: deregulation and consequences for cell behaviour. Biochim Biophys Acta 1602: 114-130

Gray MJ, Zhang J, Ellis LM, Semenza GL, Evans DB, Watowich SS, Gallick GE (2005) HIF-1 $\alpha$, STAT3, CBP/p300 and Ref-1/APE are components of a transcriptional complex that regulates Src-dependent hypoxia-induced expression of VEGF in pancreatic and prostate carcinomas. Oncogene 24: 3110-3120

Green TP, Fennell M, Whittaker R, Curwen J, Jacobs V, Allen J, Logie A, Hargreaves J, Hickinson DM, Wilkinson RW, Elvin P, Boyer B, Carragher N, Plé PA, Bermingham A, Holdgate GA, Ward WHJ, Hennequin LF, Davies BR, Costello GF (2009) Preclinical anticancer activity of the potent, oral Src inhibitor AZD0530. Mol Oncol 3: 248-261

Heffelfinger SC, Yan M, Gear RB, Schneider J, LaDow K, Warshawsky D (2004) Inhibition of VEGFR2 prevents DMBA-induced mammary tumor formation. Lab Invest 84: 989-998

Howe FA, Robinson SP, Rodrigues LM, Griffiths JR (1999) Flow and oxygenation dependent (FLOOD) contrast MR imaging to monitor the response of rat tumors to carbogen breathing. Magn Reson Imaging 17: 1307-1318

Ishizawar R, Parsons SJ (2004) c-Src and cooperating partners in human cancer. Cancer Cell 6: 209-214

Leach MO, Brindle KM, Evelhoch JL, Griffiths JR, Horsman MR, Jackson A, Jayson GC, Judson IR, Knopp MV, Maxwell RJ, McIntyre D, Padhani AR, Price P, Rathbone R, Rustin GJ, Tofts PS, Tozer GM, Vennart W, Waterton JC, Williams SR, Workman P (2005) The assessment of antiangiogenic and antivascular therapies in early-stage clinical trials using magnetic resonance imaging: issues and recommendations. Br J Cancer 92: 1599-1610

Lesslie D, Summy J, Parikh N, Fan F, Trevino J, Sawyer T, Metcalf C, Shakespeare W, Hicklin D, Ellis L, Gallick G (2006) Vascular endothelial growth factor receptor-1 mediates migration of human colorectal carcinoma cells by activation of Src family kinases. Br J Cancer 94: 1710-1717

McPhail LD, Robinson SP (2010) Intrinsic susceptibility MR imaging of chemically induced rat mammary tumors: relationship to histologic assessment of hypoxia and fibrosis. Radiology 254: 110-118

McSheehy PMJ, Weidensteiner C, Cannet C, Ferretti S, Laurent D, Ruetz S, Stumm M, Allegrini PR (2010) Quantified tumor $T_{1}$ is a generic early-response imaging biomarker for chemotherapy reflecting cell viability. Clin Cancer Res 16: 212-225

Michaelis LC, Ratain MJ (2006) Measuring response in a post-RECIST world: from black and white to shades of grey. Nat Rev Cancer 6: 409-414

Miller K, Hutchins G, Miller M, Badve S, Sledge G (2003) ZD6474-mediated vascular changes in a breast cancer xenograft model illustrated by positron emission tomography. In Proc. AACR-NCI-EORTC Conference on New Agents and Novel Targets. p B1 association with the MRC and Department of Health (England) grants C1060/A10334, CR-UK project grant C16412/A6269, NHS funding to the NIHR Biomedical Research Centre, The Royal Society and AstraZeneca Pharmaceuticals. SPR is the recipient of a Royal Society University Research Fellowship. We thank Allan Thornhill and his staff for animal care and maintenance, and Philippe Robert at Guerbet for the supply of Sinerem.

Mross K, Fasol U, Frost A, Benkelmann R, Kuhlmann J, Buchert M, Unger C, Blum H, Hennig J, Milenkova TP, Tessier J, Krebs AD, Ryan AJ, Fischer R (2009) DCE-MRI assessment of the effect of vandetanib on tumor vasculature in patients with advanced colorectal cancer and liver metastases: a randomized phase I study. J Angiogen Res 1: 5

Robinson SP, Kalber TL, Howe FA, McIntyre DJ, Griffiths JR, Blakey DC, Whittaker L, Ryan AJ, Waterton JC (2005) Acute tumor response to ZD6126 assessed by intrinsic susceptibility magnetic resonance imaging. Neoplasia 466-474

Robinson SP, Rijken PF, Howe FA, McSheehy PM, van der Sanden BP Heerschap A, Stubbs M, van der Kogel AJ, Griffiths JR (2003) Tumor vascular architecture and function evaluated by non-invasive susceptibility MRI methods and immunohistochemistry. J Magn Reson Imaging 17: $445-454$

Rusello SV, Shore SK (2004) SRC in human carcinogenesis. Front Biosci 9: 139-144

Sanderson S, Valenti M, Gowan S, Patterson L, Ahmad Z, Workman P, Eccles SA (2006) Benzoquinone ansamycin heat shock protein 90 inhibitors modulate multiple functions required for tumor angiogenesis. Mol Cancer Ther 5: 522-532

Tropres I, Lamalle L, Peoc'h M, Farion R, Usson Y, Decorps M, Remy C (2004) In vivo assessment of tumoral angiogenesis. Magn Reson Med 51: 533-541

Walker-Samuel S, Boult JKR, McPhail LD, Box G, Eccles SA, Robinson SP (2012) Non-invasive in vivo imaging of vessel calibre in orthotopic prostate tumour xenografts. Int J Cancer 130: 1284-1293

Walker-Samuel S, Orton M, McPhail LD, Robinson SP (2009) Robust estimation of the apparent diffusion coefficient (ADC) in heterogeneous solid tumors. Magn Reson Med 62: 420-429

Waterton JC, Pylkkanen L (2012) Qualification of imaging biomarkers for oncology drug development. Eur J Cancer 48: 409-415

Wedge SR, Klinowska T, Smith NR, Brown H, Farrington PM, Chang K-M South M, Graham A, Womersley L, Hall PA, Barnett S, Ogilvie DJ (2007) Examining inhibition of VEGFR and EGFR signaling in MMTV-neu transgenic mice with well-established tumors: an immunohistochemical and oncogenomic analysis of AZD2171, vandetanib and gefitinib treatment. In Proc. AACR. Abstract \#2122

Wedge SR, Ogilvie DJ, Dukes M, Kendrew J, Chester R, Jackson JA, Boffey SJ, Valentine PJ, Curwen JO, Musgrove HL, Graham GA, Hughes GD, Thomas AP, Stokes ES, Curry B, Richmond GH, Wadsworth PF, Bigley AL, Hennequin LF (2002) ZD6474 inhibits vascular endothelial growth factor signaling, angiogenesis, and tumor growth following oral administration. Cancer Res 62: 4645-4655

Winkler F, Kozin SV, Tong RT, Chae SS, Booth MF, Garkavtsev I, Xu L, Hicklin DJ, Fukumura D, di Tomaso E, Munn LL, Jain RK (2004) Kinetics of vascular normalization by VEGFR2 blockade governs brain tumor response to radiation: role of oxygenation, angiopoietin-1, and matrix metalloproteinases. Cancer Cell 6: 553-563

Workman P, Aboagye EO, Balkwill F, Balmain A, Bruder G, Chaplin DJ, Double JA, Everitt J, Farningham DA, Glennie MJ, Kelland LR, Robinson V, Stratford IJ, Tozer GM, Watson S, Wedge SR, Eccles SA, Navaratnam $\mathrm{V}$, Ryder S (2010) Guidelines for the welfare and use of animals in cancer research. Br J Cancer 102: 1555-1577

Workman P, Aboagye EO, Chung Y-L, Griffiths JR, Hart R, Leach MO, Maxwell RJ, McSheehy PMJ, Price PM, Zweit J (2006) Minimally invasive pharmacokinetic and pharmacodynamic technologies in hypothesistesting clinical trials of innovative therapies. J Natl Cancer Inst 98: 580-598

This work is published under the standard license to publish agreement. After 12 months the work will become freely available and the license terms will switch to a Creative Commons Attribution-NonCommercial-Share Alike 3.0 Unported License. 